\title{
Minimalinvasive Chirurgie bei Malignomen des Gastrointestinaltrakts: Magen - Kontra-Position
}

\author{
Katja Ott Susanne Blank Markus Büchler \\ Klinik für Allgemein-, Viszeral- und Transplantationschirurgie, Universitätsklinik Heidelberg, Deutschland
}

\author{
Schlüsselwörter \\ Laparoskopische Magenchirurgie · Vorteile $\cdot$ Nachteile
}

\section{Zusammenfassung}

Hintergrund: Bedingt durch Änderungen im Lebensstil gewinnen laparoskopische Verfahren zunehmend an Bedeutung. Zur laparoskopischen Magenchirugie existieren vor allem Studien aus Asien, wo die Inzidenz des Magenkarzinoms und besonders des Frühkarzinoms hoch ist und wo Tumoren häufig im unteren Magendrittel lokalisiert sind. Konträr verhält es sich in Deutschland mit einer rückläufigen Inzidenz des Magenkarzinoms und mit meist lokal fortgeschrittenen Tumoren proximaler Lokalisation. Methode und Ergebnisse: Die Metaanalysen enthalten überwiegend Daten zur laparoskopisch assistierten distalen Gastrektomie mit einer Inzision von 5-6 cm zum Bergen bzw. für schwierige Operationsschritte wie Schließen der Anastomose bzw. Komplettierung der Lymphadenektomie. Für das laparoskopische Vorgehen sprechen übereinstimmend der geringere intraoperative Blutverlust, die kürzere Hospitalisierungsdauer, der geringere postoperative Schmerzmittelverbrauch und das frühere Einsetzen der Darmtätigkeit - allesamt Parameter, die theoretisch auch durch den Einsatz von entsprechendem Instrumentarium und die Etablierung von standardisierten "FastTrack»-Abläufen in der offenen Chirurgie erreicht werden könnten. Bezüglich der Kosten sowie der Morbidität sind die Studien nicht eindeutig. Mit etwa 300 min bleibt die Operationsdauer signifikant länger, auch nach abgeschlossener Lernkurve von etwa 20-60 laparoskopischen Operationen pro Operateur. Dies sind Operationszahlen, die im Westen nur an wenigen Zentren realistisch erreicht werden können. Schlussfolgerungen: Onkologische Gleichwertigkeit konnte bisher nur durch eine ähnliche Anzahl entfernter Lymphknoten, nicht durch ein LangzeitFollow-up gezeigt werden. Somit ist die laparoskopische Magenchirurgie derzeit in Deutschland kein Standard.

\section{Keywords}

Laparoscopic gastrectomy · Advantages · Disadvantages

\section{Summary}

Minimally Invasive Surgery for Malignancies of the Gastrointestinal Tract: Gastrectomy - Contra Position

Background: Due to changes in life style, laparoscopic procedures are gaining more importance. For gastric cancer, mainly studies on laparoscopy-assisted distal gastrectomies from Asian countries with a high incidence of gastric cancer, and especially early gastric cancer, are available. In contrast, in Germany, the incidence of gastric cancer is decreasing and most tumors are locally advanced and localized in the proximal third. Method and Results: Meta-analyses predominantly include data on laparoscopy-assisted distal gastrectomies with an incision of 5-6 $\mathrm{cm}$, for salvage or for difficult operation steps such as closure of the anastomosis or completion of lymphadenectomy. Clear benefits of laparoscopic or laparoscopy-assisted distal gastrectomies are the significantly reduced intraoperative blood loss, the shorter duration of the hospital stay, the lower postoperative analgesic consumption, and the shorter time to the first flatus. However, all these parameters might also be reached by standardized fasttrack open surgery with modern equipment. Studies are inconclusive regarding the intra- and postoperative costs and morbidity, but with about $300 \mathrm{~min}$, the duration of laparoscopic gastric resections remains longer compared to open surgery, even after a learning curve of 20-60 laparoscopic procedures. These are operation numbers that are difficult to reach for a single surgeon in western countries, even in large clinical centers. Conclusions: The longterm oncological impact of laparoscopic resections remains unclear; only the number of harvested lymph nodes seems to be equivalent. Therefore, laparoscopic gastric surgery is currently not standard in Germany.

\begin{tabular}{ll}
\hline KARGER & @ 2013 S. Karger GmbH, Freiburg \\
Fax +49 761 452 5714 & \\
Information@Karger.com & $\begin{array}{l}\text { Accessible online at: } \\
\text { www.karger.com/vim }\end{array}$
\end{tabular}




\section{Einleitung}

Im Jahr 1994 wurde durch Kitano et al. [1] die erste laparoskopisch assistierte Billroth-I-Magenresektion in Japan beschrieben. Seither ist in Japan trotz initialer Vorbehalte aufgrund der deutlich schlechteren Tumorbiologie des Magenkarzinoms (z.B. im Vergleich zum Kolonkarzinom mit dem höheren Risiko der Entwicklung einer Peritonealkarzinose bzw. von Port-Site-Metastasen) die Zahl bis 2007 auf beeindruckende 5000 laparoskopische Eingriffe pro Jahr beim Magenkarzinom angestiegen [2]. Änderungen in der Erwartungshaltung der Patienten und verbesserte Patienteninformation führen häufig zu Diskussionen, ob Eingriffe auch schonender, d.h. laparoskopisch, mit geringeren postoperativen Schmerzen, kürzerem Krankenhausaufenthalt und kosmetisch besserem Ergebnis durchgeführt werden könnten [3]. Eine Verbesserung der Lebensqualität [3], auch beeinflusst durch die oben genannten Faktoren, wird bei identischem onkologischem Ergebnis sowohl in Studien als auch in der Bevölkerung zunehmend gefordert. Die laparoskopische Chirurgie beim Magenkarzinom hat in Deutschland derzeit einen extrem geringen Stellenwert, obwohl sie, wie durch randomisierte Studien gezeigt, technisch machbar ist $[1,4-6]$ und unter Umständen in manchen Bereichen auch Vorteile bringen könnte. Neben Lebensqualität [3] und onkologischem Ergebnis besitzen selbstverständlich auch ökonomische Aspekte wie Operationsdauer, Krankenhausliegezeit und intraoperative Kosten für das Instrumentarium zunehmend Relevanz. Bei der Analyse der Sinnhaftigkeit des laparoskopischen Vorgehens ist aber auch zu berücksichtigen, dass in Deutschland die Inzidenz des Magenkarzinoms insgesamt rückläufig ist, etwa 70\% der Magenkarzinome als lokal fortgeschrittene Tumoren diagnostiziert werden [7, 8] und ein hoher Prozentsatz der Tumoren proximal [9] lokalisiert ist. Dies sind allesamt Parameter, die ein laparoskopisches Vorgehen nicht begünstigen.

\section{Studienlage}

Insgesamt existieren sechs randomisierte kontrollierte Studien [3, 10-14] (Tab. 1), die in den meisten kürzlich publizierten Metaanalysen [15-18] eingeschlossen wurden. Die Studie von Huscher et al. [14] wurde nur zum Teil und z.B. bei Zeng et al. [15] nicht eingeschlossen, da in dieser Studie im Gegensatz zu den asiatischen Studien auch lokal fortgeschrittene Magenkarzinome einbezogen wurden. Da zum Nachweis der Effizienz einer neuen chirurgischen Technik höhere Fallzahlen erforderlich sind, wurden in allen Metaanalysen ausgewählte nicht randomisierte Studien zur Erhöhung der Fallzahl eingefügt. Die 18 bei Zeng et al. [15] zusätzlich eingeschlossenen nicht randomisierten Studien sind alle aus Asien und enthalten nur Frühkarzinome; sie beinhalten unterschiedliche Formen der Lymphadenektomie (D1 und D2) [19] und unterschiedliche Arten der Rekonstruktion (BI/BII mit oder ohne Handnaht). Die zweite hochrangig publizierte aktuelle Metaanalyse [17] aus einem westlichen Zentrum, dem Memorial Sloan-Kettering Cancer Center, enthält neben den sechs oben genannten randomisierten kontrollierten Studien 19 weitere, nicht randomisierte Studien, von denen lediglich zwei [20, 21] nicht asiatischen Ursprungs sind und ebenfalls die oben geschilderten heterogenen Parameter beinhalten. Die aufgeführten Parameter zeigen bereits, wie schwierig es ist, aus den vorliegenden Studien sinnvolle Schlüsse für westliche Patienten $\mathrm{zu}$ ziehen, da nicht nur traditionell Behandlungsunterschiede zwischen Asien und der westlichen Welt bestehen, sondern auch erhebliche Unterschiede bezüglich Tumorkategorien und heterogene Methodik bzw. Technik bei einer Empfehlung berücksichtigt werden müssten - hier seien exemplarisch laparoskopisch $[14,18]$ versus laparoskopisch assistiert [3, 10-13], BI [3, 10-12] versus BII [13] und DI versus DII $[11,14]$ genannt (Tab. 1). Aus der westlichen Welt existieren nur sehr limitierte Daten zur laparoskopischen oder laparoskopisch assistierten, distalen oder totalen Gastrektomie [5, 14, 20-22]; im Gegensatz zu Asien wurde allerdings, bedingt durch die unterschiedlichen Tumorcharakteristika, in drei $[14,21,22]$ von vier Studien eine komplett laparoskopische Gastrektomie durchgeführt. Eine totale laparoskopische Magenresektion wird übereinstimmend als eine technisch schwierige Operation eingeschätzt $[5,18]$. Die Daten, die zum Beweis der technischen Machbarkeit in einem kommunalen Haus an 49 Patienten publiziert wurden, mit einer Konversionsrate von $12 \%$, einer postoperativen Letalität von $8,2 \%$ und durchschnittlich 11 untersuchten Lymphknoten, sind ehrlich, aber ernüchternd [22].

Tab. 1. Existierende randomisierte Studien

\begin{tabular}{|c|c|c|c|c|c|c|c|c|}
\hline Autoren & Herkunftsland & $\begin{array}{l}\text { Publiziert } \\
\text { in }\end{array}$ & Studienintervall & $\begin{array}{l}\text { Einschluss- } \\
\text { kriterien }\end{array}$ & $\begin{array}{l}\text { Laparoskopisch } \\
\text { assistiert/offen }\end{array}$ & $\begin{array}{l}\text { Follow-up, } \\
\text { Monate }\end{array}$ & $\begin{array}{l}\text { Lymphaden- } \\
\text { ektomie }\end{array}$ & Rekonstruktion \\
\hline Kitano et al. [10] & Japan & 2002 & 1998-2001 & cT1 & $14 / 14$ & $24,3 / 18,8$ & D1 & BI (Handnaht) \\
\hline Huscher et al. [14] & Italien & 2005 & 1992-1996 & cT1-4, N0-2 & $30 / 29$ & 49,7 & $\mathrm{D} 1 / \mathrm{D} 2$ & ÖJ-RY/BII \\
\hline Hayashi et al. [12] & Japan & 2005 & 1999-2001 & cT1 & $14 / 14$ & $39 / 45$ & D1 & BI \\
\hline Lee und Han [11] & Korea & 2005 & 2001-2003 & cT1 & $24 / 23$ & $14 / 14$ & D2 & BI \\
\hline Kim et al. [3] & Korea & 2008 & 2003-2005 & cT1, N0-1 & $82 / 82$ & $12 / 12$ & D1/D2 & BI \\
\hline Kim et al. [13] & Korea & 2010 & 2006-2007 & cT1N0-1/cT2N0 & $179 / 163$ & $-1-$ & D1/D2 & BI/BII/ÖJ-RY \\
\hline
\end{tabular}




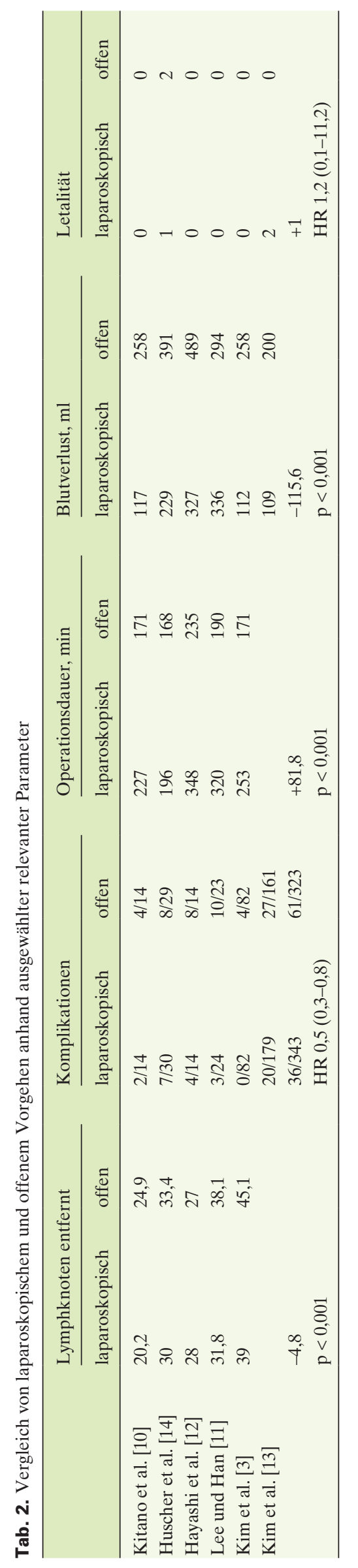

\section{Lymphadenektomie}

Jahrzehntelang wurde in Europa die Art der Lymphadenektomie kontrovers diskutiert [23-25] und es bestand aufgrund signifikant erhöhter Morbidität und einem onkologischen Benefit nur in Subgruppen [23] ein Trend zur D1-Lymphadenektomie. Seit der Präsentation der 15-Jahres-Follow-up-Daten der holländischen Magenkarzinomstudie [26] ist aber die D2Lymphadenektomie als Standard in Europa generell akzeptiert, insbesondere da meist lokal fortgeschrittene Tumorstadien vorliegen und eine radikale Lymphadenektomie erfordern $[8,27]$. Beim Frühkarzinom im asiatischen Raum wird die laparoskopische Durchführung einer D1- oder D2-Lymphadenektomie nicht standardisiert gehandhabt [3]. Eine generelle D2Lymphadenektomie für das Frühkarzinom wird aus onkologischen Gesichtspunkten in Asien nicht gefordert [19], obwohl sie technisch machbar ist [4, 6]. Die zitierten Studien aus Asien zeigen eine geringe Pankreasfistelrate, hohe Anzahlen entfernter Lymphknoten und keinen Unterschied in den entfernten Lymphknotenstationen [4, 6]. Zweifelsohne sollte in Europa bei weit mehr fortgeschrittenen Magenkarzinomen eine D2Lymphadenektomie erfolgen [26]. Westliche Daten zeigen, dass diese mit 26-30 laparoskopisch entfernten Lymphknoten mit geringer Morbidität und Mortalität möglich ist [14, 21].

Die Metaanalyse von Vinuela et al. [17] zeigt generell für die Lymphadenektomie, unabhängig von der Art (D1/D2), einen signifikanten Vorteil für die offene Gruppe mit 3,9 mehr entfernten Lymphknoten $(\mathrm{p}<0,001)$, der durch Zeng et al. [15] bestätigt wird (Tab. 2). Keine signifikanten Unterschiede zeigen sich in der Subgruppenanalyse für die Subgruppe $<$ D2 und die Subgruppe D2 [15].

Generell sollte man allerdings akzeptieren, dass die laparoskopische D2-Lymphadenektomie als technisch schwierig eingeschätzt wird und nur erfahrenen laparoskopisch arbeitenden Chirurgen vorbehalten sein sollte.

\section{Intraoperativer Blutverlust}

Übereinstimmend wird in der Literatur beschrieben, dass der intraoperative Blutverlust beim laparoskopischen Vorgehen signifikant geringer ist als bei der offenen Chirurgie [2, 15, 17] (Tab. 2).

\section{Operationsdauer}

Ebenso übereinstimmend besteht ein Konsens, dass die Operationsdauer in der laparoskopischen Magenchirurgie signifikant höher ist $[2,15,17]$. In den randomisierten Studien liegt die mittlere Dauer der laparoskopischen Operation 82 min über dem offenen Vorgehen ( $<<0,00001)$ [2] (Tab. 2), wobei die mittlere Operationsdauer bei Huscher et al. [14] für eine total laparoskopische Gastrektomie mit 196 min im Mit- 
tel extrem kurz ist. Die Angaben aus dem asiatischen Raum für die laparoskopisch assistierte distale Gastrektomie liegen dagegen zwischen 227 und 348 min mittlerer Operationsdauer [2, 3, 10-12], was mit den übrigen westlichen Studien mit 270399 min mittlerer Operationsdauer [20, 21] übereinstimmt. Es handelt sich somit um einen Eingriff, bei dem mit einer Operationsdauer von etwa $5 \mathrm{~h}$ zu rechnen ist.

\section{Postoperativer Verlauf}

\section{Schmerzmittelverbrauch}

Ein weiterer Vorteil der laparoskopischen Chirurgie ist, dass der postoperative Schmerzmittelverbrauch bei laparoskopisch magenresezierten Patienten signifikant geringer ist als bei offen operierten [15]. Dieser Aspekt bleibt ebenfalls erhalten, wenn man die D1- und D2-lymphadenektomierten Patienten getrennt analysiert [15].

\section{Krankenhausliegedauer}

Alle Studien bis auf diejenige von Kitano et al. [10] zeigten übereinstimmend eine signifikante Reduktion der Krankenhausliegedauer, durchschnittlich um 3,6 [17] bis 4,7 Tage [15], in der Subgruppe der D2-lymphadenektomierten Patienten sogar um 7,3 Tage [15]. Dabei sind die Liegezeiten vor allem in den westlichen Studien mit 5 [20, 22] bis 7 Tagen [21] beeindruckend kurz.

\section{Einsetzen der Darmtätigkeit}

Die Darmtätigkeit beginnt bei den Patienten mit laparoskopischem Vorgehen früher, auch unter Berücksichtigung der Art der Lymphadenektomie [15].

\section{Mortalität}

Die Mortalität unterscheidet sich nicht signifikant zwischen den beiden Operationsverfahren [15] (Tab. 2).

\section{Morbidität}

Die Komplikationsrate ist nach laparoskopischer Chirurgie in den Metaanalysen signifikant reduziert [11, 15, 17] (Tab. 2), wobei viele Studien beim Konfidenzintervall (CI) 1 überschreiten. Die Metaanalyse von Vinuela et al. [17] zeigt differenziert signifikant reduzierte internistische Komplikationen (relatives Risiko (RR) 0,49; CI 0,31-0,77) und chirurgische Minorkomplikationen (RR 0,62; CI 0,46-0,83), bei ähnlichem Auftreten von Majorkomplikationen (RR 0,84; CI 0,56-1,27). Die größte randomisierte koreanische Studie [13] zeigt keinen signifikanten Unterschied, allerdings war das mittlere Alter in dieser Studie 55 Jahre bei einem Body-Mass-Index von 23,5 kg/m². Das gesunde, junge Kollektiv mag der Grund sein, warum keine Unterschiede gezeigt werden konnten. Die Patienten aus westlichen Ländern sind in der Regel älter, zum Teil mit erheblichen Begleiterkrankungen und nicht so schlank. Daher könnten diese Patienten, die eine hohe Komplikationsrate bei offenen
Operationen besitzen, potenziell vom laparoskopischen Vorgehen profitieren. Ferner scheinen späte Komplikationen beim laparoskopischen Vorgehen signifikant seltener [17].

\section{Onkologische Aspekte}

Die Daten zum Langzeitüberleben sind spärlich [12, 14, 28]. Das maximale Follow-up innerhalb der randomisierten Studien aus Asien liegt bei 39 Monaten median für das laparoskopische Vorgehen [12]. Aus der westlichen Welt liegen von Huscher et al. [14] die 5-Jahres-Überlebensraten von 56\% für den offenen Chirurgiearm und von 59\% für das laparoskopische Vorgehen vor, allerdings bei limitierter Patientenzahl.

Die onkologische Gleichwertigkeit ist der wichtigste Diskussionspunkt bezüglich des laparoskopischen Vorgehens. Von den im Rahmen von randomisierten Studien laparoskopisch resezierten Frühkarzinomen in Asien existieren bei sehr begrenztem Follow-up keine signifikanten Überlebensunterschiede in Abhängigkeit von der operativen Strategie. Dies ist auch nicht zu erwarten, da die Rezidivwahrscheinlichkeit eng mit der T-Kategorie assoziiert ist [29, 30] und die Prognose von Frühkarzinomen exzellent ist.

Da die bisherigen Studien valide Überlebensdaten weitgehend schuldig bleiben, wird meistens die onkologische Qualität an der Anzahl der entfernten Lymphknoten bemessen. Die Anzahl scheint wie oben beschrieben adäquat, doch in der Gesamtanalyse werden offen signifikant mehr Lymphknoten entfernt (Tab. 2). Die weiterführende Diskussion zu lokal fortgeschrittenen Karzinomen bleibt derzeit rein theoretisch, da bis auf einige wenige Studien $[14,20,21]$ nur cT1Karzinome laparoskopisch reseziert wurden und bei diesen Studien bis auf die von Huscher et al. [14] wiederum keine Überlebensdaten existieren, sondern nur eine Beschreibung der Gleichwertigkeit der Lymphadenektomie bei extrem limitierten Fallzahlen [20, 21].

Bezüglich der onkologischen Qualität hat die laparoskopische bzw. laparoskopisch assistierte Gastrektomie bei lokal fortgeschrittenen Tumoren (cT2/3/4), bei denen auch obligat eine D2-Lymphadenektomie erforderlich ist, ihre Gleichwertigkeit im Vergleich zur offenen Chirurgie nicht gezeigt.

\section{Kosten}

Die Kosten werden von drei nicht randomisierten Studien analysiert, wobei die intraoperativen Kosten durch die Verwendung von LigaSure ${ }^{\mathrm{TM}}$ und Staplern vermeintlich beim laparoskopischen Vorgehen höher sein müssten. Zwei Studien aus Japan zeigten allerdings, dass keine Unterschiede bei den intraoperativen Kosten und den Gesamtkrankenhauskosten existieren, was wiederum überrascht, da die Liegedauer ja signifikant kürzer ist. Eine Studie aus China zeigt vermehrte intraoperative Kosten bei ähnlichen Gesamtkrankenhauskosten. 


\section{Lernkurve}

Ein in der westlichen Welt sehr wichtiger Aspekt, der in Diskussionen oft nicht adäquat berücksichtigt wird, ist die Lernkurve. Das Magenkarzinom ist im Westen relativ selten und fällt auch nicht obligat unter die Zentrumschirurgie, sodass die Fallzahl pro Operateur auch in der offenen Chirurgie relativ gering ist. Es existieren in der offenen Chirurgie Daten, dass die Fallzahl pro Operateur bei der totalen Gastrektomie signifikant mit schwerwiegenden Komplikationen assoziiert ist [31].

Die vorgegebene Zahl an erforderlichen laparoskopischen bzw. laparoskopisch assistierten Gastrektomien, um die Lernkurve abzuschließen, variiert in der Literatur zwischen 20 und 300 [11, 15, 28]. Die Zahlen bezüglich der Lernkurve für laparoskopische Magenchirurgie sind häufig auch an die Erfahrung in der offenen Chirurgie geknüpft. Abhängig von den Studien werden 23-700 offene Gastrektomien mit D2-Lymphadenektomie angegeben. Obwohl die erforderlichen Zahlen von Studie zu Studie massiv variieren, bleiben doch auch, wenn nur die Untergrenzen berücksichtigt werden, ernsthafte Bedenken, ob die erforderliche Lernkurve in Deutschland realistisch in einem adäquaten Zeitrahmen durchlaufen werden kann.

\section{Fazit}

Eine laparoskopische oder laparoskopisch assistierte Magenresektion ist technisch zweifelsohne möglich, aber selbst in Asien weit davon entfernt, ein Standard zu sein. Die aktuell vorliegende Studienlage ist relativ schlecht. Es existieren lediglich sechs randomisierte Studien, von denen diejenige von Huscher et al. [14] häufig aus den Metaanalysen ausgeschlossen wird. Die meisten Studien stammen aus Asien und beinhalten meist nur cT1-Tumoren, allerdings mit erheblicher Heterogenität bezüglich operativer Technik mit D1- oder D2Lymphadenektomie, BI- oder BII-Rekonstruktion und Vorgehen mit Stapler oder Handnaht, sodass die Ergebnisse schwer zu vergleichen sind und sich kaum definitive Schlüsse ziehen lassen. Praktisch alle Studien aus Asien beschreiben eine laparoskopisch assistierte distale Gastrektomie und keine totale laparoskopische Gastrektomie, und fast alle haben kein Langzeit-Follow-up.

Nur Frühkarzinome werden in Asien routinemäßig laparoskopisch reseziert, was sich auch in den publizierten Metaanalysen $[15,17]$ widerspiegelt. Die Datenlage für die westliche Welt ist noch wesentlich dünner [14, 20-22]. Im Gegensatz zu Asien wäre im Westen auch wesentlich häufiger eine totale Gastrektomie mit D2-Lymphadenektomie erforderlich, die laparoskopisch als technisch anspruchsvolle Operation mit höherer Komplikationsrate als eine laparoskopisch assistierte distale Gastrektomie bewertet wird [5], da Frühkarzinome sehr selten sind. Die eindeutigen Vorteile der laparoskopischen Magenchirurgie sind die kürzere Liegedauer, geringerer intraoperativer Blutverlust und geringerer postoperativer Schmerzmittelverbrauch. Diese Vorteile sind relativ schwache Parameter und könnten gegebenenfalls auch durch offene Short-Track-Strategien erreicht werden. Offen ist der Unterschied beider therapeutischer Konzepte bezüglich Letalität, Komplikationen und Kosten, wobei es beim laparoskopischen Vorgehen einen Trend zu weniger Komplikationen zu geben scheint. Onkologisch ist das laparoskopische Vorgehen seine Gleichwertigkeit noch schuldig, da es kaum Langzeitüberlebensdaten gibt, insbesondere nicht für lokal fortgeschrittene Tumoren. Außerdem ist eine nicht unerhebliche Lernkurve für jeden einzelnen Operateur zu durchlaufen, die bei den geforderten Zahlen in Deutschland zu einem realistischen Problem werden könnte.

Entsprechend der aktuellen und geschilderten Datenlage ist die laparoskopische Magenresektion in Deutschland derzeit somit kein Standard.

\section{Disclosure Statement}

Es bestehen keine Interessenkonflikte.

\section{Literatur}

1 Kitano S, Iso Y, Moriyama M, Sugimachi K: Laparoscopy-assisted Billroth I gastrectomy. Surg Laparosc Endosc 1994;4:146-148.

2 Kodera Y, Fujiwara M, Ohashi N, Nakayama G, Koike M, Morita S, Nakao A: Laparoscopic surgery for gastric cancer: a collective review with meta-analysis of randomized trials. J Am Coll Surg 2010;211:677-686.

3 Kim YW, Baik YH, Yun YH, Nam BH, Kim DH, Choi IJ, Bae JM: Improved quality of life outcomes after laparoscopy-assisted distal gastrectomy for early gastric cancer: results of a prospective randomized clinical trial. Ann Surg 2008;248:721-727.
4 Katai H, Sasako M, Fukuda H, Nakamura K, Hiki N, Saka M, Yamaue H, Yoshikawa T, Kojima K: Safety and feasibility of laparoscopy-assisted distal gastrectomy with suprapancreatic nodal dissection for clinical stage I gastric cancer: a multicenter phase II trial (JCOG 0703). Gastric Cancer 2010;13:238-244.

5 Lee SE, Ryu KW, Nam BH, Lee JH, Kim YW, Yu JS, Cho SJ, Lee JY, Kim CG, Choi IJ, Kook MC, Park SR, Kim MJ, Lee JS: Technical feasibility and safety of laparoscopy-assisted total gastrectomy in gastric cancer: a comparative study with laparoscopy-assisted distal gastrectomy. J Surg Oncol 2009;100:392-395.
6 Song KY, Kim SN, Park CH: Laparoscopy-assisted distal gastrectomy with D2 lymph node dissection for gastric cancer: technical and oncologic aspects. Surg Endosc 2008;22:655-659.

7 Siewert JR, Böttcher K, Stein HJ, Roder JD: Relevant prognostic factors in gastric cancer: ten-year results of the German Gastric Cancer Study. Ann Surg 1998;228:449-461.

8 Meyer L, Steinert R, Nowak L, Gellert K, Ludwig K, Saeger D, Gastinger I, Lippert H: Prospektive Multizenterstudie zur Chirurgie des Magenkarzinoms - Ein Beitrag zur klinischen Versorgungsforschung. Zentralbl Chir 2005;130:97-105. 
9 Siewert JR, Feith M, Stein HJ: Biologic and clinical variations of adenocarcinoma at the esophago-gastric junction: relevance of a topographic-anatomic subclassification. J Surg Oncol 2005;90:139-146, discussion 146.

10 Kitano S, Shiraishi N, Fujii K, Yasuda K, Inomata $\mathrm{M}$, Adachi Y: A randomized controlled trial comparing open vs laparoscopy-assisted distal gastrectomy for the treatment of early gastric cancer: an interim report. Surgery 2002;131:S306-S311.

$\checkmark 11$ Lee JH, Han HS: A prospective randomized study comparing open vs laparoscopy-assisted distal gastrectomy in early gastric cancer: early results. Surg Endosc 2005;19:168-173.

12 Hayashi H, Ochiai T, Shimada H, Gunji Y: Prospective randomized study of open versus laparoscopy-assisted distal gastrectomy with extraperigastric lymph node dissection for early gastric cancer. Surg Endosc 2005;19:1172-1176.

13 Kim HH, Hyung WJ, Cho GS, Kim MC, Han SU, Kim W, Ryu SW, Lee HJ, Song KY: Morbidity and mortality of laparoscopic gastrectomy versus open gastrectomy for gastric cancer: an interim report a phase III multicenter, prospective, randomized trial (KLASS trial). Ann Surg 2010;251:417-420.

14 Huscher CG, Mingoli A, Sgarzini G, Sansonetti A Di Paola M, Recher A, Ponzano C: Laparoscopic versus open subtotal gastrectomy for distal gastric cancer: five-year results of a randomized prospective trial. Ann Surg 2005;241:232-237.

15 Zeng YK, Yang ZL, Peng JS, Lin HS, Cai L: Laparoscopy-assisted versus open distal gastrectomy for early gastric cancer: evidence from randomized and nonrandomized clinical trials. Ann Surg 2012;256:39-52.

16 Sun J, Li J, Wang J, Pan T, Zhou J, Fu X, Zhang S Meta-analysis of randomized controlled trials on laparoscopic gastrectomy vs. open gastrectomy for distal gastric cancer. Hepatogastroenterology 2012; 59:1699-1705.
17 Vinuela EF, Gonen M, Brennan MF, Coit DG, Strong VE: Laparoscopic versus open distal gastrectomy for gastric cancer: a meta-analysis of randomized controlled trials and high-quality nonrandomized studies. Ann Surg 2012;255:446-456.

18 Bracale U, Rovani M, Bracale M, Pignata G, Corcione F, Pecchia L: Totally laparoscopic gastrectomy for gastric cancer: meta-analysis of short-term outcomes. Minim Invasive Ther Allied Technol 2012;21:150-160.

19 Yoshikawa T, Tsuburaya A, Kobayashi O, Sairenji M, Motohashi H, Noguchi Y: Is D2 lymph node dissection necessary for early gastric cancer? Ann Surg Oncol 2002;9:401-405.

20 Strong VE, Devaud N, Allen PJ, Gonen M, Brennan MF, Coit D: Laparoscopic versus open subtotal gastrectomy for adenocarcinoma: a case-control study. Ann Surg Oncol 2009;16:1507-1513.

21 Guzman EA, Pigazzi A, Lee B, Soriano PA, Nelson RA, Benjamin Paz I, Trisal V, Kim J, Ellenhorn JD: Totally laparoscopic gastric resection with extended lymphadenectomy for gastric adenocarcinoma. Ann Surg Oncol 2009;16:2218-2223.

22 Stotland PK, Chia S, Cyriac J, Hagen JA, Klein LV: Safe implementation of laparoscopic gastrectomy in a community-based general surgery practice. Surg Endosc 2009;23:356-362.

23 Hartgrink HH, van de Velde CJ, Putter H, Bonenkamp JJ, Klein Kranenbarg E, Songun I, Welvaart K, van Krieken JH, Meijer S, Plukker JT, van Elk PJ, Obertop H, Gouma DJ, van Lanschot JJ, Taat CW, de Graaf PW, von Meyenfeldt MF, Tilanus H, Sasako M: Extended lymph node dissection for gastric cancer: who may benefit? Final results of the randomized Dutch gastric cancer group trial. J Clin Oncol 2004:22:2069-2077.
4 Bonenkamp JJ, Songun I, Hermans J, et al: Randomised comparison of morbidity after D1 and D2 dissection for gastric cancer in 996 Dutch patients. Lancet 1995;345:745-748.

25 Cuschieri A, Weeden S, Fielding J, Bancewicz J, Craven J, Joypaul V, Sydes M, Fayers P: Patient survival after D1 and D2 resections for gastric cancer: long-term results of the MRC randomized surgical trial. Surgical Co-operative Group. Br J Cancer 1999;79:1522-1530.

26 Songun I, Putter H, Kranenbarg EM, Sasako M, van de Velde CJ: Surgical treatment of gastric cancer: 15-year follow-up results of the randomised nationwide Dutch D1D2 trial. Lancet Oncol 2010; 11:439-449.

27 Siewert JR, Bottcher K, Roder JD, Busch R, Hermanek P, Meyer HJ: Prognostic relevance of systematic lymph node dissection in gastric carcinoma. German Gastric Carcinoma Study Group. Br J Surg 1993;80:1015-1018.

28 Lee J, Kim W: Long-term outcomes after laparoscopy-assisted gastrectomy for advanced gastric cancer: analysis of consecutive 106 experiences. J Surg Oncol 2009;100:693-698.

29 Mikami K, Maekawa T, Shinohara T, Hoshino S, Yamauchi Y, Noritomi T, Yamashita Y: Predictive factors of early recurrent death after a curative resection of gastric cancer. Int Surg 2009;94:144-148.

30 D'Angelica M, Gonen M, Brennan MF, Turnbul AD, Bains M, Karpeh MS: Patterns of initial recurrence in completely resected gastric adenocarcinoma. Ann Surg 2004;240:808-816.

31 Fujita T, Yamazaki Y: Influence of surgeon's volume on early outcome after total gastrectomy. Eur J Surg 2002;168:535-538. 